Check for updates

Cite this: Nanoscale Horiz., 2019, 4,735

Received 24th October 2018 Accepted 31st January 2019

DOI: $10.1039 / c 8 n h 00377 g$

rsc.li/nanoscale-horizons

\section{Enhanced electrochemical biosensor and supercapacitor with 3D porous architectured graphene via salt impregnated inkjet maskless lithography $\dagger$}

\author{
John A. Hondred, (D) ${ }^{a}$ Igor L. Medintz (D) ${ }^{b}$ and Jonathan C. Claussen (D)*a
}

\begin{abstract}
Advances in solution-phase graphene patterning has provided a facile route for rapid, low-cost and scalable manufacturing of electrochemical devices, even on flexible substrates. While graphene possesses advantageous electrochemical properties of high surface area and fast heterogenous charge transport, these properties are attributed to the edge planes and defect sites, not the basal plane. Herein, we demonstrate enhancement of the electroactive nature of patterned solution-phase graphene by increasing the porosity and edge planes through the construction of a multidimensional architecture via salt impregnated inkjet maskless lithography (SIIML) and $\mathrm{CO}_{2}$ laser annealing. Various sized macroscale pores ( $<25$ to $\sim 250 \mu \mathrm{m}$ ) are patterned directly in the graphene surface by incorporating porogens (i.e., salt crystals) in the graphene ink which act as hard templates for pore formation and are later dissolved in water. Subsequently, microsized pores ( $100 \mathrm{~nm}$ to $2 \mu \mathrm{m}$ in width) with edge plane defects are etched in the graphene lattice structure by laser annealing with a $\mathrm{CO}_{2}$ laser, simultaneously improving electrical conductivity by nearly three orders of magnitude (sheet resistance decreases from $>10000$ to $\sim 50 \Omega \mathrm{sq}^{-1}$ ). We demonstrate that this multidimensional porous graphene fabrication method can improve electrochemical device performance through design and manufacture of an electrochemical organophosphate
\end{abstract} biosensor that uses the enzyme acetylcholinesterase for detection. This pesticide biosensor exhibits enhanced sensitivity to acetylthiocholine compared to graphene without macropores $\left(28.3 \mu \mathrm{AnM}^{-1}\right.$ to $13.3 \mu \mathrm{A} \mathrm{nM}^{-1}$ ) and when inhibited by organophosphate pesticides (paraoxon) has a wide linear range (10 $\mathrm{nM}$ to $500 \mathrm{nM}$ ), low limit of detection $(0.6 \mathrm{nM})$, and high sensitivity $\left(12.4 \mathrm{nA} \mathrm{nM}^{-1}\right)$. Moreover, this fabrication method is capable of patterning complex geometries [i.e. interdigitated electrodes (IDEs)] even on flexible surfaces as demonstrated by an IDE supercapacitor made of SIIML graphene on a heat

\footnotetext{
${ }^{a}$ Department of Mechanical Engineering, Iowa State University, Ames, IA, 50011, USA. E-mail: jcclauss@iastate.edu; Tel: +515-294-4690

${ }^{b}$ U.S. Naval Research Laboratory, Center for Biomolecular Science \& Engineering, Washington DC, 20375, USA

$\dagger$ Electronic supplementary information (ESI) available. See DOI: 10.1039/ c8nh00377g
}

\section{Conceptual insights}

A new manufacturing method called Salt Impregnated Inkjet Maskless Lithography (SIIML) is introduced and shown to enhance the electrochemical activity of patterned solution-phase graphene. Salt crystals mixed into the graphene ink act as hard templates or porogens for the subsequent creation of macroscale pores. After a solvent-based graphene liftoff process defines the printed graphene electrodes, a $\mathrm{CO}_{2}$ laser removes the salt crystals to reveal the pores $(100 \mathrm{~nm}-2 \mu \mathrm{m})$ and to increase the electrical conductivity of the graphene $\left(\sim 50 \Omega \mathrm{sq}^{-1}\right)$. This three-dimensional hierarchy/architecture provides increased edge plane and defects in the graphene, which improves the catalytic activity and capacitance of patterned graphene. This work demonstrates how the patterned graphene can be functionalized to create a highly sensitive pesticide biosensor and high power/energy density electrochemical supercapacitor. While other researchers have also shown that increasing the surface area of graphene can improve electrochemical activity, few researchers have been able to demonstrate a manufacturing method to pattern high surface area graphene; for those who have, the material typically suffers from low conductivity. In addition, these high surface area, conductive graphene circuits could act as a platform technology for batteries, biofuel cells, electrochemical biosensors, and dye-sensitized solar cells as well as flexible and heat sensitive substrates for wearable technology.

sensitive polymer substrate. The supercapacitor demonstrates a high energy density of $0.25 \mathrm{~mW} \mathrm{~h} \mathrm{~cm}^{-3}$ at a power density of $0.3 \mathrm{~W} \mathrm{~cm}$. These electrochemical devices demonstrate the benefit of using SIIML and $\mathrm{CO}_{2}$ laser annealing for patterning graphene electrodes with a multidimensional porous surface even on flexible substrates and is therefore a platform technology which could be applied to a variety of different biosensors and other electrochemical devices.

\section{Introduction}

Graphene has emerged as one of the most attractive electrocatalytic transduction materials due to its extraordinary electrical and thermal conductivity, high mechanical strength, biocompatibility, high carrier capacity/mobility, and potential 
high surface area. ${ }^{1-3}$ Specifically, electrodes comprising graphene and graphene derivatives (graphene nanoparticles, reduced graphene oxides, oxidized graphene, functionalized graphene) have demonstrated high electrical conductivity and/or catalytic capability. For example, in the past decade graphene has been used for electrode materials in electrochemical devices such as supercapacitors, ${ }^{4}$ batteries, ${ }^{5}$ fuel cells, ${ }^{6}$ cell electrode stimuli ${ }^{7,8}$ and sensors. ${ }^{9,10}$

Graphene is typically produced by chemical vapor deposition (CVD) which can yield nearly pristine (defect-free) single or few layer 2D sheets. ${ }^{11}$ In the context of electrochemistry, these 2D pristine sheets or basal plane geometry exhibit four orders of magnitude lower specific capacitance and seven orders of magnitude lower electron transfer rates than the edges planes or edge defect sites of the graphene. ${ }^{12}$ Numerous methods have been used to increase the electrochemical surface area (ECSA) of graphene. For example, nanosphere lithography using selfassembly of polystyrene, ${ }^{13}$ silica, ${ }^{14}$ or $\mathrm{MnO}_{2}{ }^{15}$ nanospheres has been shown to etch nanopores into CVD grown graphene to increase the defect density in the lattice structure (more edge planes) and consequently improved the sensitivity of electrochemical sensors. Others have constructed three-dimensional CVD grown graphene foam out of a nickel scaffold, ${ }^{16}$ polystyrene colloidal particles as a sacrificial template, ${ }^{17}$ as well as various other manufacturing methods derived from chemical vapor deposition, hydrothermal methods, and sugar-blowing production. ${ }^{18}$ While these methods significantly increase the ECSA of the graphene electrode, they often require clean room technology, and/or high temperature fabrication. Such complex fabrication protocols are hence expensive and not amenable to high-throughput manufacturing such as roll-to-roll fabrication.

Solution-phase graphene provides an attractive alternative to costly CVD grown graphene as it can be synthesized from low-cost graphite with mechanical/chemical exfoliation. ${ }^{19,20}$ Various methods have been exploited to increase the ECSA of solution-phase graphene. For example, the "breath-figure" method uses moist gas that condenses on organic solution-phase graphene which results in the formation of highly controlled evaporated water droplet pores $(\sim 2 \mu \mathrm{m}) .^{21,22}$ Others have electrochemically reduced graphene oxide which provides a simple, one-step procedure to create $3 \mathrm{D}$ porous graphene coatings with pores ranging between $1-10 \mu \mathrm{m}$ between graphene flakes. ${ }^{16}$ Colloidal nanoparticles were used as template scaffolding which produced a hollow free-standing 3D graphene structure $(500 \mathrm{~nm}) .{ }^{23}$ While each of these methods provide effective means for creating a highly porous $3 \mathrm{D}$ graphene architecture, they do not provide facile routes to pattern the solution-phase graphene into electrodes and in many cases are not stable (i.e., the architectures collapse in the air). ${ }^{16}$

Simple, low-cost methods such as inkjet, ${ }^{24}$ aerosol, ${ }^{25}$ and screen printing ${ }^{26}$ provide simple fabrication methods for patterning solution-phase graphene into highly defined graphene electrodes; however, these graphene surfaces typically have little ECSA as graphene flakes are tightly compact exposing predominately their basal planes. ${ }^{27}$ Various techniques have been employed to alter the graphene flake orientation, for example, Choi et al., incorporated nafion polymers into the graphene solution which made a hydrophobic (contact angle $\sim 161^{\circ}$ ) rough surface, however, the graphene film lacked conductivity $\left(>10 \mathrm{k} \Omega \mathrm{sq}^{-1}\right) .{ }^{28}$ Hersam and coworkers used polymer-phase inversion to tailor the porosity of graphene, similarly, the increase in porosity lead to a decrease in conductivity ( $\sim 1000 \mathrm{~S} \mathrm{~m}^{-1}$ at $15 \%$ glycerol $) .{ }^{29}$ Alternatively, we have demonstrated that secondary post-processing methods such as laser annealing can significantly increase the conductivity $\left(\sim 100 \Omega \mathrm{sq}^{-1}\right)^{10}$ while simultaneously enhancing the electroactive surface area of graphene by nano/micro structuring pores into the graphene by orientating superficial graphene flakes vertically. ${ }^{10,30,31}$ However, these methods do not make macrosized pores in the graphene surface or micropores in the graphene lattice structure while retaining electrical conductivity. Macropores in the graphene surface facilitate enhanced diffusion of substrates towards and away from the active sites (defect sites and edge planes in the graphene). Therefore, adding the additional three-dimensional hierarchy/architects of different pore sizes, as exhibited in these developed electrodes for example, has been shown to result in higher electroactive surface area and enhanced catalytic activity. ${ }^{32,33}$

Herein we demonstrate a facile manufacturing process of patterning solution-phase graphene electrodes with high electroactive surface area utilizing different orders of pore sizes via salt impregnated inkjet maskless lithography (SIIML) and $\mathrm{CO}_{2}$ laser annealing. This technique modifies the newly developed IML method for rapid prototyping highly defined graphene films by incorporating salt crystals as porogens to increase the ECSA. ${ }^{10,31}$ Sieved salt powders have been demonstrated in the past to act as efficient porogens for $3 \mathrm{D}$ printed resins, ${ }^{34-36}$ polymer-nanotube scaffolds for bone formation ${ }^{37}$ and porous carbon powders for gas absorbance. ${ }^{38}$ Similarly, we demonstrate that salt crystals can act as a hard template porogens for macrosize $(<25$ to $\sim 250 \mu \mathrm{m})$ defects in $2 \mathrm{D}$ thin film electrochemical devices. We also demonstrate for the first time, the use of a $\mathrm{CO}_{2}$ laser to anneal patterned solution-phased graphene which etches microsize $(\sim 100 \mathrm{~nm}-2 \mu \mathrm{m})$ pores in the graphene surface. This high ECSA graphene surface displayed enhanced electrochemical performance as demonstrated by an electrochemical pesticide biosensor with high sensitivity (12.4 $\left.\mathrm{nA} \mathrm{nM}^{-1}\right)$ and low limit of detection $(0.6 \mathrm{nM})$ to paraoxon (a model organophosphate pesticide). Furthermore, we show that this multidimensional porous graphene surface is a platform technology that can be applied to electrochemical devices beyond biosensors, including energy storage, as demonstrated by an electrochemical supercapacitor fabricated via the SIIML process which portrays high energy and power density $\left(0.25 \mathrm{~mW} \mathrm{~h} \mathrm{~cm}^{-3}\right.$ at $\left.0.3 \mathrm{~W} \mathrm{~cm}^{-3}\right)$.

\section{Methods and materials}

\subsection{Chemicals and reagents}

Completely chemically reduced single layer graphene oxide (referred to henceforth as graphene) was purchased from 
ACS Materials (GN1P0005). Sodium chloride ( $\mathrm{NaCl}$ ) was purchased from Sigma-Aldrich, ground using mortar and pestle, and filtered into different sizes with 8 in wire cloth sieves. Acetylcholinesterase (ACHE) from Electrophorus electricus was purchased from Sigma Aldrich as lyophilized powder (200-1000 units per mg) and was used without any further purification. ACHE ink aliquots were made by dissolving the enzyme ( $2 \mathrm{mg}$ ) in $1 \mathrm{~mL}$ of $50 \%$ glycerol and $50 \%$ 1x phosphate-buffer solution (0.1 M PBS, pH 7.0), aliquoted into $25 \mu \mathrm{L}$, and stored in a $-80{ }^{\circ} \mathrm{C}$ freezer until use. All pesticides (including paraoxon) were obtained from Chem Service. All other chemicals and solvents were purchased from Sigma-Aldrich and were used without any other purification unless otherwise stated. Water was filtered through a B-Pure Water Purification system (resistivity, $18.2 \mathrm{M} \Omega \mathrm{cm}^{-2}$ ).

\subsection{Electrode fabrication}

2.2.1 Printing graphene via salt impregnated inkjet maskless lithography (SIIML). Graphene electrodes were manufactured by modifying the inkjet maskless lithography (IML) method published earlier. ${ }^{10,31}$ A highly concentrated graphene ink was fabricated by probe sonicated $100 \mathrm{mg}$ ethylcellulose, $100 \mathrm{mg}$ nitrocellulose, and $200 \mathrm{mg}$ graphene in a solution of $25 \mathrm{~mL}$ terpineol and $200 \mathrm{~mL}$ of acetone for 4 hours, followed by boiling off the acetone. Ethylcellulose and nitrocellulose have been shown to help suspend the graphene flakes in solvents, improve the adhesion of the graphene to the surface, and carbonize upon annealing which reducing flake to flake resistance. ${ }^{27}$ Sieved microsized salt crystals $(<25$ to $\sim 250 \mu \mathrm{m})$ were then incorporated into the graphene ink which made macrosized pores into the patterned graphene surface, henceforth referred to as salt impregnated inkjet maskless lithography (SIIML). In summary, a sacrificial polymer layer (20\% formaldehyde resin and $80 \%$ cyclohexanone) was inkjet printed using a Dimatix Material Printer (DMP-2850, 10 pL nozzles) onto heat treated polyethylene terephthalate (PET, [kemafoil, Coveme TSL W]). A highly concentrated graphene ink was spin-coated (1000 rpm for $30 \mathrm{~s}$ ) over the patterned sacrificial layer and postbaked for 10 min under a heat gun (Steinel \#HB1750K) at a distance of 18 inches. An additional layer of graphene ink was then spin-coated over the sample that included discrete-sized salt crystals (ground using mortar/pestle and sieved into distinct sizes, 25-50 $\mu \mathrm{m}$ unless noted otherwise). The graphene ink was typically used immediately, but only slight settling of salt was observed after a week and was easily vortex mixed back to a suspension for re-use. The substrate was then again postbaked to remove solvents and increase graphene adhesion, followed by impinging the entire surface with an acetone wash bottle to remove the sacrificial layer. This process created a highly defined electrode pattern with salt crystals impregnated within the surface.

2.2.2 $\mathrm{CO}_{2}$ laser annealing. The SIIML graphene electrodes were laser annealed using a $\mathrm{CO}_{2}$ laser engraver (Orion Motor Tech $40 \mathrm{~W}$ ). The raster engraving rate was set to $350 \mathrm{~mm} \mathrm{~s}^{-1}$ for all experiments, and the power of the engraver was set to $6.2 \%$ (estimated at $6.2 \mathrm{~W}$ ) with a spot size $\sim 150 \mu \mathrm{m}$, unless otherwise specified. These settings were found to properly anneal the graphene (carbonize surfactants and "weld" flakes together $)^{9}$ which increased conductivity. Using a $\mathrm{CO}_{2}$ laser to anneal and etch the surface of the graphene revealed the salt crystals, facilitating the "opening" of the macropore (Fig. S1, $\mathrm{ESI} \dagger$ ). Using a $\mathrm{CO}_{2}$ laser also etched the individual graphene flakes making microsized pores $(100 \mathrm{~nm}-2 \mu \mathrm{m})$ in graphene surface which further enhanced the catalytic abilities by providing additional edge defects (Fig. S2, ESI $\dagger$ ).

\subsection{Biosensor design and fabrication}

2.3.1 ACHE ink preparation and deposition. SIIML electrodes were functionalized with ACHE using 1-ethyl-3-(3-dimethylaminopropyl) carbodiimide and $N$-hydroxysuccinimide (EDC/NHS) chemistry, which facilitated the functionalization of carboxyl groups to the amines within the ACHE enzyme. ${ }^{39}$ In summary, the $\mathrm{CO}_{2}$ laser annealed SIIML electrodes were dipped in a solution of EDC/NHS mixture (50 mM EDC, $50 \mathrm{mM}$ NHS) and incubated for $30 \mathrm{~min}$ on a tilter mixer. The electrodes were then rinsed with 1x PBS, excess water was blown off but not completely dried as the surface remained still "wetted", this allowed for easier pipetting onto the slightly hydrophobic graphene surface. Next, $5 \mu \mathrm{L}$ of the pre-aliquoted ACHE solution was immediately pipetted onto the surface and incubated overnight. Electrodes were washed 3 times in 1x PBS to remove any unbound ACHE before testing. Note for final inhibition pesticide sensor, a diluted concentration (97\%) of ACHE was immobilized on the electrode surface which provides a higher inhibition signal output. This is because less enzyme coverage equates to lower concentrations of pesticides that are needed to inhibit said enzyme and hence change the biosensor signal output. ${ }^{40}$

2.3.2 Electro-analytical measurements. All electrochemical biosensor measurements and procedures were conducted on a $\mathrm{CH}$ instrument potentiostat (600E series) with a standard three electrode setup (single membrane $\mathrm{Ag} / \mathrm{AgCl}$ reference electrode, platinum wire counter electrode, and $\mathrm{CO}_{2}$ laser annealed SIIML graphene working electrode). Unless specified, each test was performed with $15 \mathrm{~mL}$ of 1x PBS buffer. For pesticide sensing, the biosensor was first tested in buffer to achieve baseline and ensure that solution did not contain contaminates that oxidize at $400 \mathrm{mV}$. The sensor was then tested in $5 \mathrm{mM} \mathrm{ACTH} \mathrm{to}$ acquire a base sensitivity to ACTH. The biosensor was then placed into the aqueous test solution with the pesticide for a minimum of $500 \mathrm{~s}$. During this incubation step, the pesticide diffuses to and permanently binds to ACHE, preventing it from catalyzing ACTH and therefore the product thiocholine from oxidizing on the electrode surface. The sensor is then rinsed with buffer and retested in the same $5 \mathrm{mM}$ ACTH to acquire the decrease in amperometric response (referred to henceforth as inhibition sensitivity), see representative amperometric graph (Fig. S3, ESI $\dagger$ ).

\subsection{Supercapacitor design and fabrication}

2.4.1 SIIML graphene supercapacitors fabrication. Graphene supercapacitors were designed similar to the SIIML graphene biosensor transduction layer, however, the rotation rate of the spin-coating process was increased from $1000 \mathrm{rpm}$ to $2000 \mathrm{rpm}$ 
so a thinner layer of graphene $(\sim 1.5 \mu$ m per layer $)$ was deposited. A thinner layer $(3 \mu \mathrm{m}$, verified through ion milling through sample and measuring cross-sectional height using SEM) was used in order to increase the overall power and energy density by using a smaller supercapacitor volume. A dry gel electrolyte was used for the supercapacitor consisting of poly(vinyl alcohol) and phosphoric acid $\left(\mathrm{PVA} / \mathrm{H}_{3} \mathrm{PO}_{4}\right) .{ }^{29}$ In Short, $6 \mathrm{~mL}$ of DI water was mixed with $3 \mathrm{~mL}$ isopropol alcohol (IPA) and $1 \mathrm{~mL}$ concentrated $\mathrm{H}_{3} \mathrm{PO}_{4}$. The solution was then placed on a hotplate $\left(80{ }^{\circ} \mathrm{C}\right)$ and $1 \mathrm{~g}$ PVA (poly(vinyl alcohol)) was slowly added until completely dissolved. A $25 \mu \mathrm{L}$ aliquot of the solution was then pipetted onto each of the IDEs, which were placed in a dessicator for one hour to remove any air bubbles and insure that the electrolyte properly wetted the porous graphene electrode. Finally, the electrodes were dried in ambient air overnight.

2.4.2 Electrochemical supercapacitor characterization. The SIIML and IML (no salt) graphene supercapacitors were characterized by cyclic voltammetry (CV) and galvanostatic charge-discharge (potentiometry) expermiments with two electrode (shorting counter and reference) setup. ${ }^{41}$ Capacitance at different current densities were calculated using the discharge curves obtained from galvanostatic graphs and eqn (1).

$$
C=\frac{I \times \Delta t}{\Delta U}
$$

where $I$ is the applied current (amps), $\Delta t$ is the discharge time (s), and $\Delta U$ is the discharge voltage after self-discharge (IR drop of the electrode) is removed. Similarly aerial surface area capacitance (eqn (2)) and volumetric capacitance (eqn (3)) are calculated by dividing the capacitance by the aerial surface area $(A=$ $\left.0.25 \mathrm{~cm}^{2}\right)$ and the total volume of the IDE $\left(V=0.000075 \mathrm{~cm}^{3}\right)$, this includes active electrode area and space between electrodes.

$$
\begin{aligned}
& C_{\mathrm{A}}=\frac{C}{A} \\
& C_{\mathrm{V}}=\frac{C}{V}
\end{aligned}
$$

The volumetric energy density $\left(E_{\mathrm{v}}\right)$ and power density $\left(P_{\mathrm{V}}\right)$ were calculated using eqn (4) and (5).

$$
\begin{gathered}
E_{\mathrm{V}}=\frac{C_{\mathrm{V}} \times \Delta U^{2}}{2 \times 3600 \times V} \\
P_{\mathrm{V}}=\frac{C_{V} \times \Delta U^{2}}{2 \times V \times \Delta t}
\end{gathered}
$$

\subsection{Electrochemical surface area (ECSA) calculations}

All ECSA experiments were conducted in $5 \mathrm{mM}$ ferri/ferrocyanide in 1x PBS. CVs were conducted with three different scan rates $\left(25,50\right.$, and $\left.100 \mathrm{mV} \mathrm{s}^{-1}\right)$ and were used to calculate the ECSA using the Randle-Sevcik equation (eqn (6)), ${ }^{42,43}$ refer to representative graphs, Fig. S4 (ESI $\dagger$ ).

$$
I_{\mathrm{p}}=2.69 \times 10^{5} A D^{1 / 2} n^{3 / 2} v^{1 / 2} C
$$

where $A$ is the effective electroactive surface area $\left(\mathrm{cm}^{2}\right), D$ is the diffusion coefficient $\left(7.6 \times 10^{-6} \mathrm{~cm}^{2} \mathrm{~s}^{-1}\right), n$ is the number of electrons in the Faradaic reaction $(n=1), v$ is the scan rate $\left(\mathrm{V} \mathrm{s}^{-1}\right), C$ is the concentration of the bulk redox species $(5 \mathrm{mM})$, and $I_{\mathrm{p}}$ is the current at the oxidation peak. Note: the width between the anodic and cathodic peak often increased with higher scan rates which is often seen in very porous materials. ${ }^{10}$

\subsection{Field emission SEM images}

Field emissions scanning electron microscopy (FESEM) images were acquired using a FEI Quanta 250 FESEM. All images were magnified to $500 \times, 1500 \times 5000 \times$, or $15000 \times$ using backscattering electrons, spot size of 3.0, and with a $10 \mathrm{kV}$ potential. A $2 \mathrm{~nm}$ conductive coating of iridium was evenly coated over all samples with a turbo-pump sputter coater to improve surface imaging by preventing surface charging and hinders electrons from penetrating into the carbon material leading to poor surface contrast.

\section{Results and discussion}

\subsection{Biosensor Design and Optimization}

3.1.1 Overview of SIIML of graphene electrode fabrication. Highly electroactive graphene transduction electrodes were fabricated by a modification to the newly developed IML process ${ }^{10,31}$ which utilizes solution-phase graphene instead of CVD grown graphene eliminating the need for high temperature annealing $\left(>1000{ }^{\circ} \mathrm{C}\right)$; the latter is costly and requires silicon wafer substrates which are insufficient for large scale roll-to-roll production. ${ }^{44}$ This manufacturing method, coined salt impregnated inkjet maskless lithography (SIIML), incorporates salt crystal as porogens in the graphene ink during IML manufacturing creating macrosized pores in the graphene. Additionally, $\mathrm{CO}_{2}$ laser annealing is employed which creates microsized pores in the surface of the graphene.

First, a polymer sacrificial layer is inkjet printed onto a disposable, low-cost, and flexible substrate (PET), Fig. 1a. A highly concentrated graphene ink is then spin-coated over

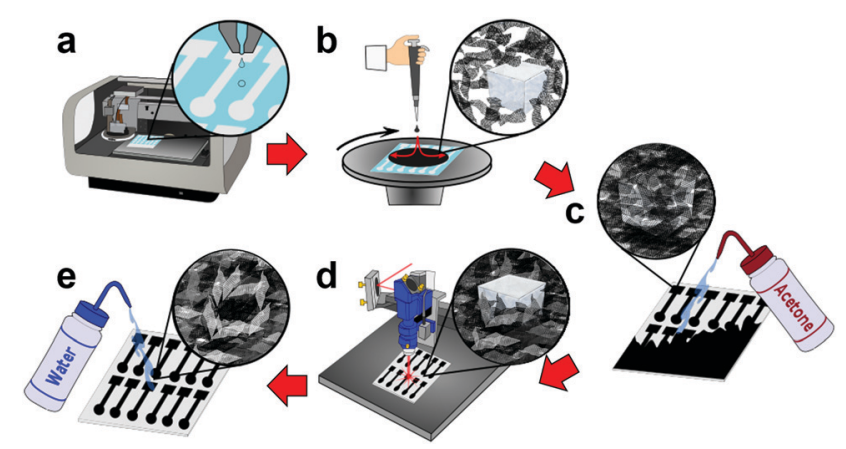

Fig. 1 Schematic diagram depicting the fabrication process steps of SIIML graphene electrodes. (a) A sacrificial polymer layer is inkjet printed as the negative of the final pattern. (b) Concentrated graphene ink impregnated with salt crystals is spin-coated. (c) The entire surface is impinged with acetone to remove the sacrificial layer, patterning the graphene. (d) $\mathrm{A} \mathrm{CO}_{2}$ laser anneals the graphene which increases conductivity, etches micropores, and reveals the salt crystals. (e) Electrodes are finally rinsed with water to remove the salt porogen, making macrosized pores within the graphene surface. 
the entire polymer sacrificial layer, followed by an additional graphene ink layer impregnated with salt crystals. The entire sample is then post-baked with a heat-gun to remove solvents and increase graphene adhesion to the substrates, Fig. 1b. As the salt crystals are ionic solids, they do not ionize in the nonpolar organic solvent and when patterned form a hard template (porogen) for macrosized pores. The entire graphene surface is then impinged by an acetone wash bottle which removes the underlying polymer sacrificial layer, patterning a highly-defined graphene electrode, Fig. 1c and Movie S1 (ESI $\dagger$ ). As acetone is not highly polar, the salt remains in its crystalline form as a hard template beneath the graphene surface. The graphene is then treated with a $\mathrm{CO}_{2}$ laser which anneals the surface, removes remaining solvents, carbonizes surfactants, and "welds" graphene flakes together. ${ }^{9}$ The $\mathrm{CO}_{2}$ laser also etches micropores into the graphene surface enhancing electrochemical activity by producing additional edge planes in the graphene surface. Furthermore, laser annealing also etches the graphene surface revealing the salt crystals, opening the surface for pore formation, Fig. 1d and Fig. S1 (ESI $\dagger$ ). Finally, the electrode is rinsed with DI water which removes the salt porogen crystals, forming macrostructured pores in the graphene surface, Fig. 1e and Movie S2 (ESI $\dagger$ ). These macropores improve electrochemical sensing by providing increased analyte diffusion rates and increased ECSA.

3.1.2 Overview of pesticide biosensor mechanism and fabrication. An electrochemical pesticide biosensor was designed in order to evaluate the enhancement in electrochemical activity of SIIML graphene and to refine manufacturing properties (i.e. laser annealing power, salt size, and salt concentration). The SIIML graphene electrodes were biofunctionalized with the enzyme ACHE via EDC/NHS chemistry, Fig. 2a. As previously mentioned and discussed in more details later, laser annealing graphene in ambient air conditions creates a high ECSA but also provides increased defect/holes in the graphene lattice structure which are rich in oxygen functional groups (epoxide sites, hydroxyl, carboxyl, etc.). The carboxylic groups provide unique immobilization sites for functionalization via EDC/NHS deprotonation mechanisms. ${ }^{45}$

This immobilization strategy (EDC/NHS) was chosen as it has been widely studied, covalently crosslinks with "zero order" length to carboxylic functional groups, is water soluble (no prior organic solvent dissolution necessary) and provides excellent subsequent stability to enzymes. ${ }^{46}$ First, EDC reacts with carboxyl groups (formed through laser annealing) to make an $O$-acylisourea intermediate ester. Second, to increase stability of this short-lived ester, NHS is added to form a stable aminereactive NHS ester, which increases immobilization efficiency of EDC coupling reaction. ${ }^{47}$ Finally, ACHE is then bound to the ester by a free amine in the enzyme, Fig. 2a.

The use of ACHE for inhibited pesticide biosensing has been demonstrated many times in the literature, but for clarity, Fig. 2b illustrates its basic mechanism. The biosensor operates by hydrolyzing the molecule ACTH which produces acetic acid and thiocholine. Two thiocholine molecules are oxidized at the graphene surface at an applied potential of $400 \mathrm{mV}$, Fig. S5 (ESI $\dagger$ ); this reaction produces dithiocholine and two electrons. The thiocholine oxidation reaction is used as a baseline amperometric signal for sensing the inhibition of ACHE by pesticides. As increasing concentrations of pesticides are added to the solution, more ACHE is inhibited which prevents the formation of thiocholine, consequently this reduces the oxidation and amperometric current (see representative amperometric graphs in Fig. S3, ESI $\dagger$ ).

3.1.3 Laser annealing process. Herein, we demonstrate for the first-time laser annealing solution-phase printed graphene with a $\mathrm{CO}_{2}$ laser. Similar to our previously published work with a Nd:YAG ${ }^{9,48}$ and diode laser, ${ }^{10,31}$ annealing the SIIML graphene with a $\mathrm{CO}_{2}$ laser also significantly increased the conductivity by "welding"' individual graphene flakes together and carbonizing

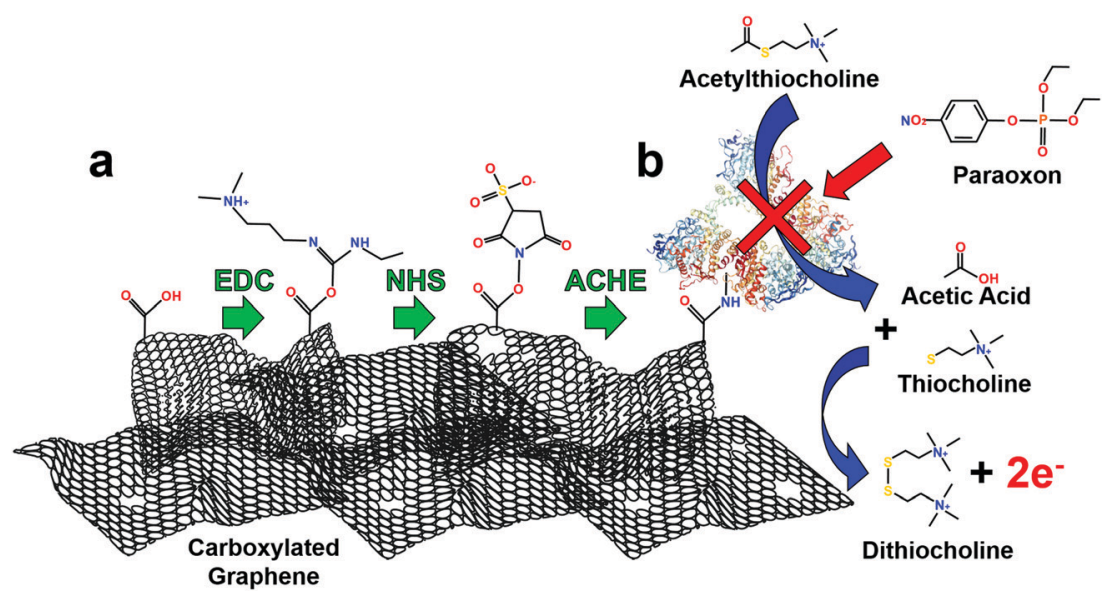

Fig. 2 Schematic diagram of ACHE biosensor depicting the functionalization strategy for pesticide biosensing using EDC/NHS. (a) From left to right: EDC reacts with carboxyl groups to make an $\mathrm{O}$-acylisourea intermediate ester. NHS reacts to form a stable amine-reactive ester. Finally, NHS ester binds ACHE to graphene surface through available amines in the enzyme. (b) Basic operations of ACHE pesticide biosensor. From top to bottom: ACTH reacts with $\mathrm{ACHE}$ and produces acetic acid and thiocholine. Thiocholine $(2 \times)$ is oxidized by the graphene electrode and produces dithiocholine and two electrons. Paraoxon (model pesticide/organophosphate) inhibits ACHE and prevents the catalysis of ACTH. Note: size not to scale. 

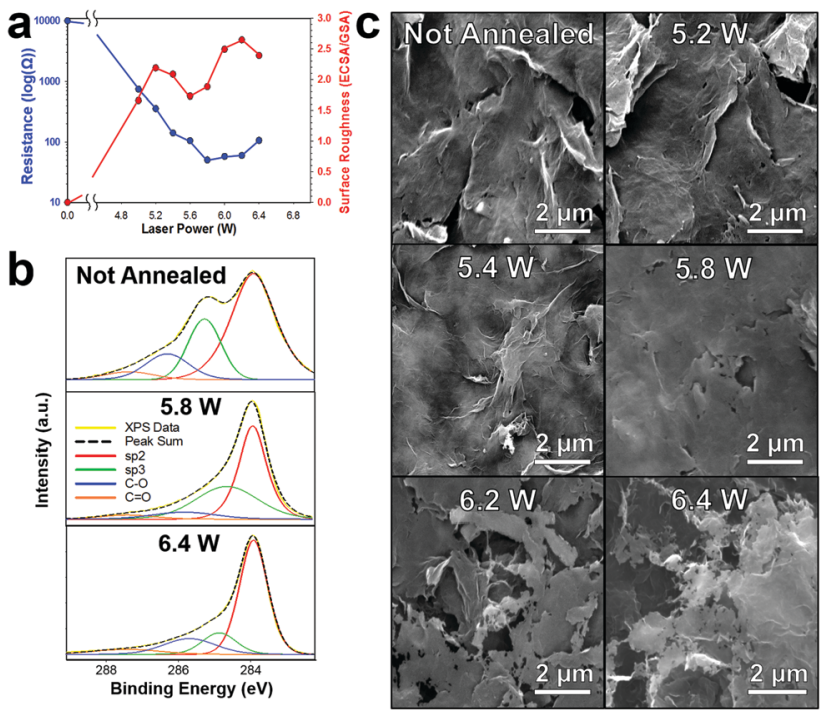

Fig. 3 Effects of $\mathrm{CO}_{2}$ laser annealing graphene. (a) Electrode sheet resistance (blue) and electrochemical surface roughness (red) versus increasing laser power. (b) XPS of not annealed, $5.8 \mathrm{~W}$, and $6.4 \mathrm{~W}$ laser annealed graphene. (c) Representative SEM of laser annealed graphene surfaces with various powers showing the initial welding of graphene flakes at low power and then etching through the surface making microsized pores at higher powers.

the surfactants (ethyl/nitrocellulose) which reduces flake-toflake resistance. The power of the $\mathrm{CO}_{2}$ laser was incrementally increased from $5 \%$ to $6.4 \%$ (estimated as $5-6.4 \mathrm{~W}$ ) which significantly lowered the sheet resistance of the graphene nearly 3 orders of magnitude ( $>10000 \Omega \mathrm{sq}^{-1}$ to $\sim 50 \Omega \mathrm{sq}^{-1}$ ) (Fig. 3a (blue)). We further confirm the carbonizing of the surfactants by XPS which shows a decrease in the $\mathrm{sp}^{3}$ peak (green) which we attribute to ethyl/nitrocellulose, Fig. 3b. Interestingly, unlike the diode laser or the Nd:YAG, ${ }^{9,10,31}$ the $\mathrm{CO}_{2}$ laser does not alter the graphene flake orientation; rather, it fuses graphene flakes together $(5 \mathrm{~W}$ till $5.8 \mathrm{~W})$ increasing the conductivity ( $\sim 10 \mathrm{k} \Omega \mathrm{sq}^{-1}$ to $\sim 50 \Omega \mathrm{sq}^{-1}$, Fig. 3a (blue)), then etches through the surface making microsize pores (Fig. 3c). The surface roughness (electrochemical surface area/geometric surface area) of the laser annealed graphene begins to increase (Fig. 3a (red)) as the resistance of the electrode decreases. The not annealed patterned graphene initially has a negligible surface roughness and high electrical resistance. When laser annealing at a power of $5.2 \mathrm{~W}$ the graphene surface roughness increased to 2.2. However, as the laser power increased past $5.2 \mathrm{~W}$ the graphene flakes begin to "weld" together making a flat plane which decreases the ECSA (surface roughness of 1.7 at $5.6 \mathrm{~W}$ ) due to decreases in available edge planes. Further increasing the laser power not only "welds" the graphene flakes together promoting even higher conductivity but starts to etch through the basal planes of the graphene which leads to increased edge plane defects by making microsize pores $(100 \mathrm{~nm}-2 \mu \mathrm{m})$ in the graphene surface and consequently increasing the electrochemical surface roughness (2.6 at a power of $6.2 \mathrm{~W}$ ). The conductivity and surface area both decrease after $6.2 \mathrm{~W}$ as the graphene is etched through the surface and the substrate begins to degrade. Therefore, for all further experiments the $\mathrm{CO}_{2}$ laser was set to $6.2 \mathrm{~W}$ with a $350 \mathrm{~mm} \mathrm{~s}^{-1}$ raster rate.

Raman spectroscopy was employed to analyze the atomic structure of the printed graphene and the effect of $\mathrm{CO}_{2}$ laser annealing. Fig. S6 (ESI $\dagger$ ) displays the Raman spectra collected from not annealed and increasing laser power annealed graphene (5.2-6.4 W) with a backscattering geometry, $1064 \mathrm{~nm}$ Nd:YAG laser and spot size of $1 \mathrm{~mm}$. Note that the spectra has been standardized so all samples have consistent $\mathrm{G}$ peak heights. The not annealed printed graphene displayed distinct $\mathrm{D}$ and $\mathrm{G}$ peaks which are characteristic for graphene and graphitic samples. However, the sharp 2D peak, typically associated with graphene is not visible. The loss of the $2 \mathrm{D}$ peak is probably due to the high concentration of surfactants within the printed graphene sample and possible impurities/functional groups on the graphene surface. However, as the laser power increases from $5.2 \mathrm{~W}$ to $6.2 \mathrm{~W}$ the $2 \mathrm{D}$ peak develops, indicating the formation of a graphene like structure by energetic photon irradiation. Furthermore, as the laser intensity increases, there is a decrease in $\left(I_{\mathrm{D}} / I_{\mathrm{G}}\right)$ ratio (from 0.72 for not annealed graphene to 0.17 for $6.2 \mathrm{~W}$ ) which suggests that the printed graphene forms a higher degree in edge-induced defects, which are conducive towards enhanced electrochemical reactivity. ${ }^{9,49}$ Additionally as the laser power increases there is an increase in $\left(I_{2 \mathrm{D}} / I_{\mathrm{G}}\right)$ ratio (from 0.23 for not annealed graphene to 0.78 for annealed graphene with a laser power of $6.2 \mathrm{~W}$ ) which correlates with reported multilayer graphene structures. ${ }^{50}$ At a laser power of $6.4 \mathrm{~W}$, the ratios reverse as the samples are etching through the surface and there are possible formation of van der Waals bonds into more a graphitic nature. In summary, the emergence of a symmetric 2D peak with an increase in $\left(I_{\mathrm{G}} / I_{\mathrm{D}}\right)$ and $\left(I_{2 \mathrm{D}} / I_{\mathrm{G}}\right)$ ratio at higher laser powers demonstrates the carbon surface is more nanostructured or nanotextured with a higher amount of edge defects and the $\mathrm{sp}^{2}$ honeycomb lattice of the surface becomes more favorable to classification as multilayered graphene.

It is interesting that we do not see a significant increase in graphene oxidation (Fig. 3b) as we are laser annealing in ambient oxygen atmosphere as seen by our previous work with a diode laser. ${ }^{31}$ Instead there is approximately a consistent small oxide layer formed across all laser powers. These oxide functional groups are most likely superficial as oxidizing deep into the graphene electrode would decrease the electrode conductivity (not seen in Fig. 3a), which may partially explain the lower sheet resistance of $\mathrm{CO}_{2}$ laser annealing $\left(\sim 50 \Omega \mathrm{sq}^{-1}\right)$ compared to using a diode laser $\left(\sim 100 \Omega \mathrm{sq}^{-1}\right) \cdot{ }^{10}$ In any case, the oxidation groups and defects in the graphene surface provides effective functionalization locations for both biomolecules or even nanoparticle nucleation locations, as we have shown previously. ${ }^{9,10,31}$

3.1.4 Salt impregnation process. The ECSA of the graphene electrodes was enhanced by incorporating macrosized pores via salt crystals in the graphene ink. Distinct sized salt crystals (ground using mortar and pestle and sieved into different sizes) were incorporated directly into the second layer of spin-coated graphene ink. Once dried, these salt crystals acted as porogens (hard templates) for macrosized pore formations in the graphene 
surface, and were subsequently removed by washing with DI water, (Movie S2, ESI $\dagger$ ). Inks with various size salt crystals (100-250 $\mu \mathrm{m}, 75-100 \mu \mathrm{m}, 50-75 \mu \mathrm{m}, 25-50 \mu \mathrm{m}$, and $<25 \mu \mathrm{m}$, based on sieve mesh size) were made and cast with IML to better characterize the microstructuring and electrochemical enhancement of SIIML. The surfaces were imaged with SEM, Fig. 4a, displaying the distinct pore structure which is controlled by the size of the salt crystals used. An electrode with no salt incorporated into the second spin-coated layer was used as a control sample and exhibit a relatively smooth surface with limited surface structures (apart from micropores formed from the laser annealing), Fig. 4a and Fig. S7 (ESI $\dagger$ ). As the salt crystal size decreases, a denser pore array is formed due to a higher salt suspension stability of the ink and the salt is able to form a tighter array when spin-coated on the surface.

This tighter pore array as well as the increased "surface-tovolume" effect (smaller pores will have higher surface area per vacancy ratio) results in an increase in surface area as the size of the pores decrease, Fig. $4 \mathrm{~b}$ (red). This effect is clearly seen by an over $50 \%$ increase in ECSA of electrodes made with $25-50 \mu \mathrm{m}$ pores a
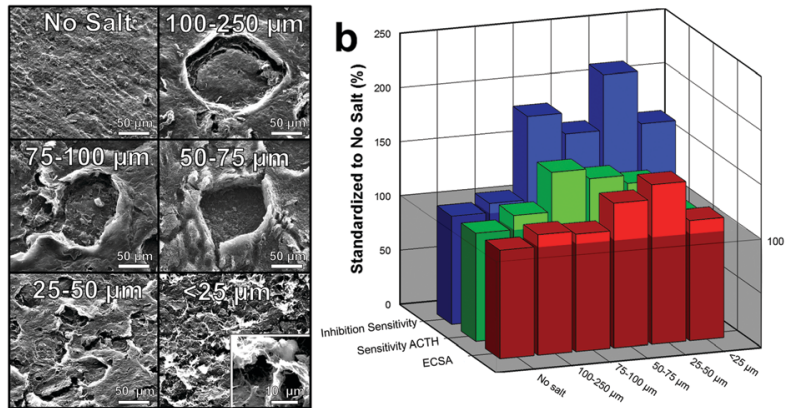

Fig. 4 Effects of various size salt crystals for macroscale pore formation. (a) SEM depicting various size salt pores made with decreasing size salt crystals. Bottom right: Magnification of $<25 \mu \mathrm{m}$ pore. (b) Graph demonstrated the effects of different size salt crystals to ECSA (red), sensitivity to $5 \mathrm{mM} \mathrm{ACTH}$ (green), and inhibition sensitivity due to $1 \mu \mathrm{M}$ paraoxon (blue). Note: graph has been standardized to no salt pores for comparison. compared to electrodes made without salt pores. Interesting, after the pore size decrease below $25 \mu \mathrm{m}$, there is a significant decrease in ECSA. While it is not completely understood, we speculate that the size is approaching the microporous size made by laser annealing and is therefore mitigating the effect of one or the other. Additionally, this decrease in ECSA could be from the decrease in perpendicular diffusion of substrate into the electrode which would decrease the electroactivity of the substrate.

Increasing the ECSA of the graphene by incorporating pores significantly enhanced the electroactivity of the electrode by providing additional sites for oxidation or reduction of the substrate. ${ }^{9}$ Moreover, the higher surface area of the porous graphene also provides additional binding locations for enzymes immobilization, which allow for improved substrate diffusion kinetics and consequently enhanced catalysis. ${ }^{10}$ Both of these effects improve the performance of the biosensor which increases its sensitivity to the analyte of interest. To test this, the electrodes were placed in a concentrated solution $(5 \mathrm{mM})$ of $\mathrm{ACTH}$ with a working potential of $400 \mathrm{mV}$ (versus $\mathrm{Ag} / \mathrm{AgCl}$ ). When ACTH reacts with ACHE, thiocholine is produced which oxidizes at the electrode. The corresponding increase in current due to the sensitivity to ACTH was graphed, Fig. $4 \mathrm{~b}$ (green). As the size of the pores decrease and the ECSA increases, there is a general increase in sensitivity to ACTH as expected. Variations from this trend $(150 \%$ for $75-100 \mu \mathrm{m}$ and $133 \%$ for $25-50 \mu \mathrm{m})$, are likely due to manufacturing errors (enzyme loading differences), experimental setup (sensors did not full equalize to baseline), or random/systematic errors. These results are further demonstrated by SIIML graphene biosensor with $25-50 \mu \mathrm{m}$ being over 2 times more sensitive to the ACHE than without salt pores, Fig. 4b (blue). For all further experiments, 25-50 $\mu \mathrm{m}$ salt particles were used as they exhibited the highest ECSA and was the most sensitive to paraoxon.

The concentration of the 25-50 $\mu \mathrm{m}$ salt/graphene ink suspension was varied to improve the pore array density of the graphene surface, Fig. 5a. As the salt concentration increased, the pores in the graphene surface on average come closer together forming a denser pore array. At $50 \mathrm{mg} \mathrm{mL}{ }^{-1}$, the pores
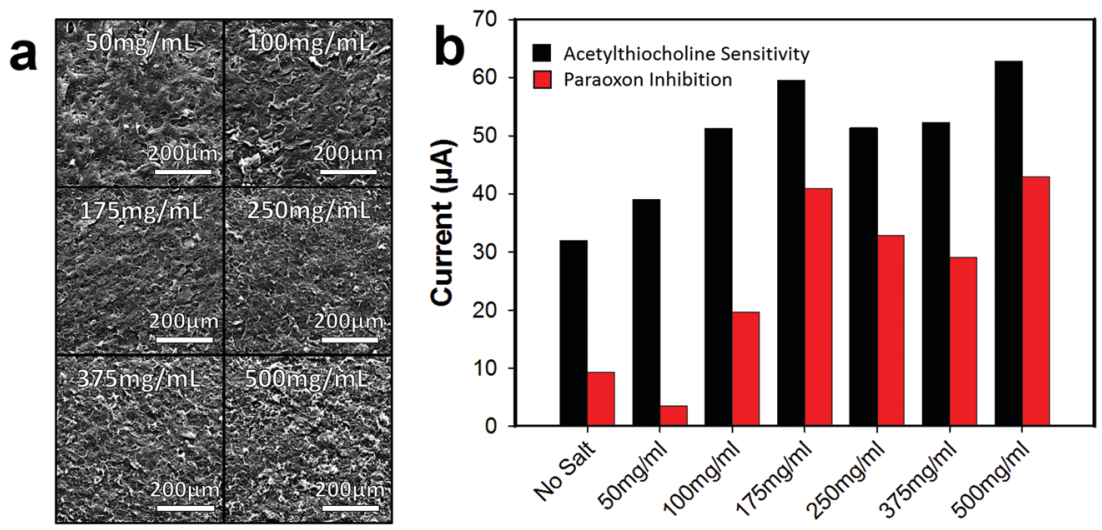

Fig. 5 Effects of various salt concretions in graphene ink. (a) Representative SEM depicting concentrations of salt forming sparse to dense pore arrays in the graphene. (b) Graph demonstrated the effects of different concentrations of salt for sensitivity to $5 \mathrm{mM} \mathrm{ACHE}$ (Black), and inhibition sensitivity due to $1 \mathrm{mM}$ paraoxon (Red). 
are fairly far apart, and a sparse array is formed (roughly estimated by visibly counting pores in SEM, $\sim 75-125$ pores per $\mathrm{cm}$ ). When the concentration was increased to $250 \mathrm{mg} \mathrm{mL}^{-1}$ a network of pores are formed $(\sim 300-500$ pores per $\mathrm{cm})$, and at $500 \mathrm{mg} \mathrm{mL}^{-1}$ a very dense array of pores is present $(\sim 500-750$ pores per $\mathrm{cm})$. Note that as the concentration of salt increases and the salt packs closer together, the pore structures are harder to distinguish due to overlap. Additionally, as the salt concentration increases, the viscosity of the graphene ink also increases. At $500 \mathrm{mg} \mathrm{mL}^{-1}$, the ink is much more viscous (making it more difficult to spin-coat) and so a thicker layer of graphene may have been applied which results in the salt not all lying flat against the bottom surface causing salt porogen overlap, Fig. S7 (ESI $\dagger$ ).

As the pore structure in the graphene becomes denser, the biosensor electrode (functionalized with ACHE), becomes more sensitive to ACTH (Fig. 5b), however, it appears that after $175 \mathrm{mg} \mathrm{mL}^{-1}$, the anodic current relatively plateaus. Interesting, this trend is not completely observed with the inhibition sensitivity of paraoxon and after $175 \mathrm{mg} \mathrm{mL}^{-1}$ the sensitivity begins to decrease. This observation is not easily explained, but we speculate that the higher surface area increased the loading of the enzyme on the graphene, resulting in more enzyme that needed to be inhibited. As the concentration of $175 \mathrm{mg} \mathrm{mL}^{-1}$ yielded a porous graphene biosensor with the most sensitivity to ACTH and the signal response was most inhibited by paraoxon, all further experiments were conducted with this salt concentration.

\subsection{Biosensor performance characterization}

The electrochemical activity of SIIML graphene electrodes was demonstrated by manufacturing a pesticide biosensor using
ACHE and refined parameters shown previously $\left(6.2 \mathrm{~W} \mathrm{CO}_{2}\right.$ laser annealing, 50-75 $\mu \mathrm{m}$ salt pores, and $175 \mathrm{mg} \mathrm{mL}^{-1}$ salt concentration). First, an array $(4 \times 10)$ of $3 \mathrm{~mm}$ diameter disk electrodes were manufactured using the procedure outlined in the Overview SIIML Graphene Electrode Fabrication (Fig. 6a). A lacquer was then pipetted onto the stem of the electrode to isolate the working area ( $3 \mathrm{~mm}$ diameter disk) and biofunctionalization was carried out as described in the Overview Pesticide Biosensor Fabrication. The ACHE pesticide sensor was placed in a saturated solution ( $5 \mathrm{mM})$ of ACTH and amperometric current was recorded with changing potentials (Fig. 6b). When the potential reached $200 \mathrm{mV}$ (versus $\mathrm{Ag} / \mathrm{AgCl}$ ) there was an increase in anodic current due to the oxidation of thiocholine. This oxidation current increases as the applied potential approaches $400 \mathrm{mV}$ then plateaus, therefore, an applied potential of $400 \mathrm{mV}$ was chosen for the ACHE biosensor. This observation is in good agreement with $\mathrm{CV}$ scans that show an oxidation peak around $400 \mathrm{mV}$ (Fig. S5, ESI $\dagger$ ) and similarly reported in the literature. $^{51,52}$

ACHE pesticide biosensors rely on the inhibition of the enzyme (paraoxon irreversibly binds to ACHE which prevents ACTH from catalyzing and hence thiocholine from oxidizing). Therefore, to test the amount of time required for organophosphates to diffuse to the electrode surface and bind to ACHE, we incubated the sensors in $1 \mu \mathrm{M}$ of paraoxon and recorded the decrease in amperometric oxidation current (which correlates directly with the percent of ACHE inhibited), Fig. 6c. After 100 seconds, there is roughly $50 \%$ inhibition of the sensor with large standard deviations; however, after 500 seconds, there is about $90 \%$ inhibition with low standard deviation and minimal
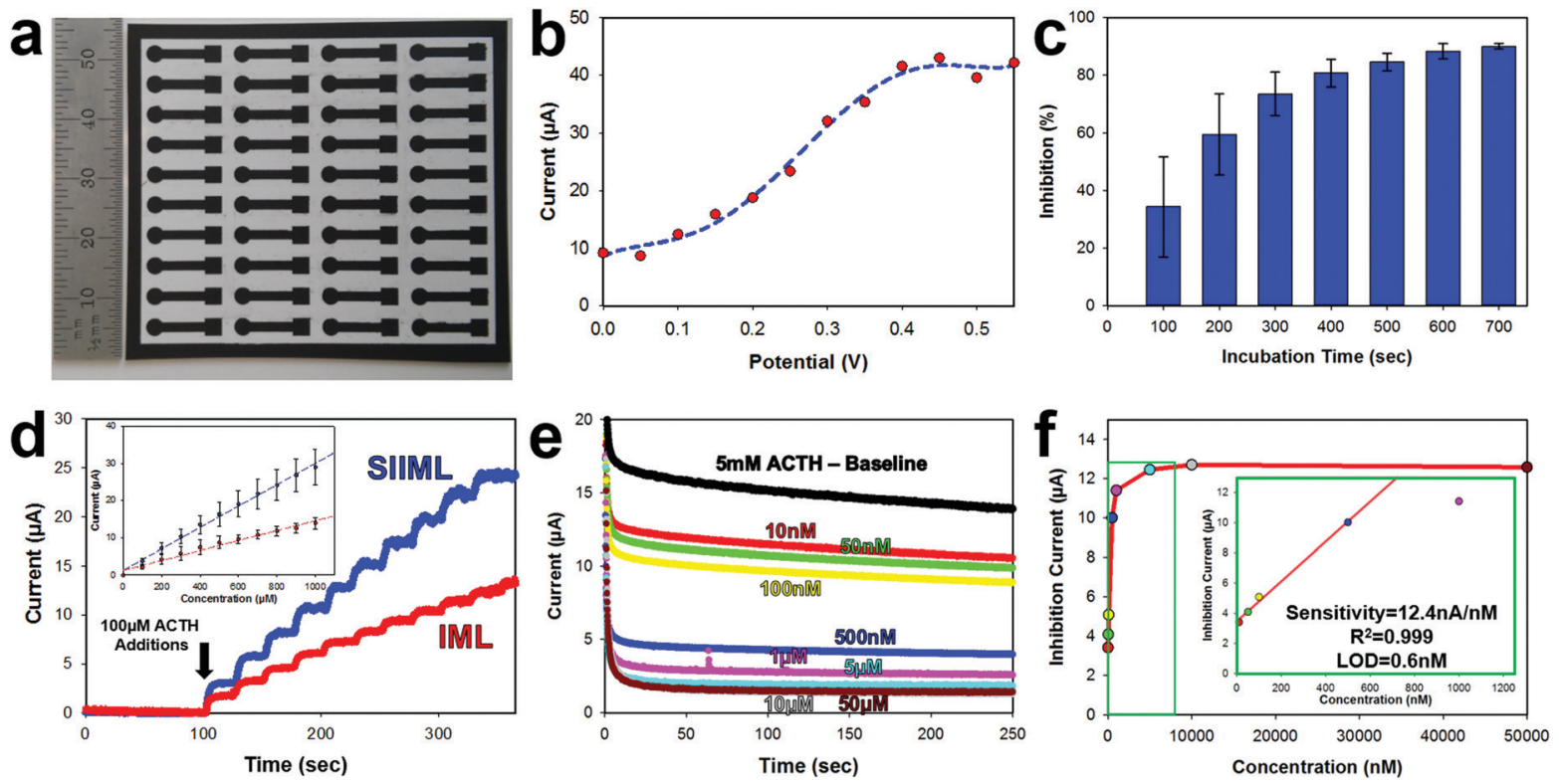

Fig. 6 ACHE pesticide biosensor characterization. (a) Photograph of patterned array $(4 \times 10)$ SIIML graphene electrodes on flexible PET substrate. (b) Effect of applied potential to amperometric response of $1 \mathrm{mM} \mathrm{ACTH}$. (c) Plot of inhibition (\%) according to incubation time. (d) Sensitivity to ACTH for SIIML (blue) and IML (red), inset: current versus concentration. (e) Amperograms of pesticide ACHE biosensor after incubating with various concentrations of paraoxon. Buffer baseline (black), $10 \mathrm{nM}$ (red), $50 \mathrm{nM}$ (green), $100 \mathrm{nM}$ (yellow), $500 \mathrm{nM}$ (blue), $1 \mu \mathrm{M}$ (pink), $5 \mu \mathrm{M}$ (aqua blue), $10 \mu \mathrm{M}$ (grey), $50 \mu \mathrm{M}$ (brown). (f) Corresponding current versus concentration, inset: magnification showing linear sensing range. 
change over the next couple of hundred seconds. Therefore, an incubation time of 500 seconds was used as a minimal time for sensor operation.

Graphene electrodes were made with (SIIML) and without salt pores (IML) for comparison. Increasing concentrations of ACTH (100 $\mu \mathrm{M}$ additions) were spiked into a buffer solution and the amperometric anodic current was recorded for both SIIML and IML. The electrodes containing salt pores had a sensitivity to ACTH of $28.3 \mu \mathrm{A} \mathrm{nM}^{-1}$ while the electrodes without salt pores had a sensitivity of $\sim 13.3 \mu \mathrm{AnM}^{-1}$, Fig. $6 \mathrm{~d}$.

Finally, the SIIML were tested for pesticide sensing with paraoxon (a model organophosphate pesticide). It should be noted here that a diluted concentration of ACHE was applied to these pesticide biosensors ( $\sim 2 \mathrm{U}$ per sensor) as having a smaller concentration of enzyme results in higher sensitivity due to less enzyme that needs to be inhibited. ${ }^{40}$ An amperometric baseline of eight different SIIML ACHE pesticide biosensors were recorded in $5 \mathrm{mM}$ ACTH which had an anodic current of $13.84 \pm 0.3 \mu \mathrm{A}$. Each of the sensors were then placed in different concentrations of paraoxon $(10 \mathrm{nM}-50 \mu \mathrm{M})$ and incubated for a minimum of $5 \mathrm{~min}$, and then placed back in the same $5 \mathrm{mM}$ ACTH solution (Fig. 6e). The concentration versus inhibition current (decrease in anodic current) was graphed (Fig. 6f) and showed a linear region up to $500 \mathrm{nM}$ and then a plateau region shortly thereafter. The sensitivity of the linear region was calculated to be $12.4 \mathrm{nA} \mathrm{nM}^{-1}$ with a limit of detection of $0.6 \mathrm{nM}$ (LOD $=3$ noise per signal, noise defined as standard deviation of three electrodes without ACHE).
Therefore, our sensor has a limit of detection below the tolerable drinking water equivalence level of the United States and Canada EPA (24 nM and $170 \mathrm{nM}$, respectively). ${ }^{53,54}$ There are many reported ACHE biosensors with lower limit of detection, even as low as $0.4 \mathrm{pM} ;{ }^{55}$ however, these biosensors often employ complex methods to enhance their sensitivity and reduce noise such as metal nanoparticles, ${ }^{56,57}$ multilayer carbon nanotubemodified glassy carbon electrodes, ${ }^{55}$ co-enzymes, ${ }^{58}$ flow cells ${ }^{55,59}$ or microelectrodes. ${ }^{60}$

\subsection{Supercapacitor performance characterization}

To further demonstrate the enhanced electrochemical activity of SIIML electrodes, supercapacitors were designed. Supercapacitors offer promising energy storage opportunities for on-chip devices as they can be fabricated directly in line with the electronics. ${ }^{61}$ Supercapacitors store energy at the interface of the electrode and electrolyte; therefore, increasing the ECSA and conductivity of the electrode improves the efficiency and capacitance of the supercapacitor. ${ }^{62}$ Interdigitated electrodes (IDEs) were designed and patterned using the procedures outlined in the Overview SIIML Graphene Electrode Fabrication, and an array $(2 \times 4)$ of IDEs were patterned, Fig. 7a. The SIIML graphene IDEs were then coated with $\mathrm{PVA} / \mathrm{H}_{3} \mathrm{PO}_{4}$ gel which acts as a dry gel electrolyte. The performance of the SIIML graphene supercapacitor was first examined with CVs of various scan rates (50 $\mathrm{mV} \mathrm{s}^{-1}$ - black to $1000 \mathrm{mV} \mathrm{s}^{-1}$ - orange), Fig. $7 \mathrm{~b}$. The CVs show a rectangular shape at low scan rates and only begin to distort in shape at very high scan rates $\left(1000 \mathrm{mV} \mathrm{s}^{-1}\right)$ due to
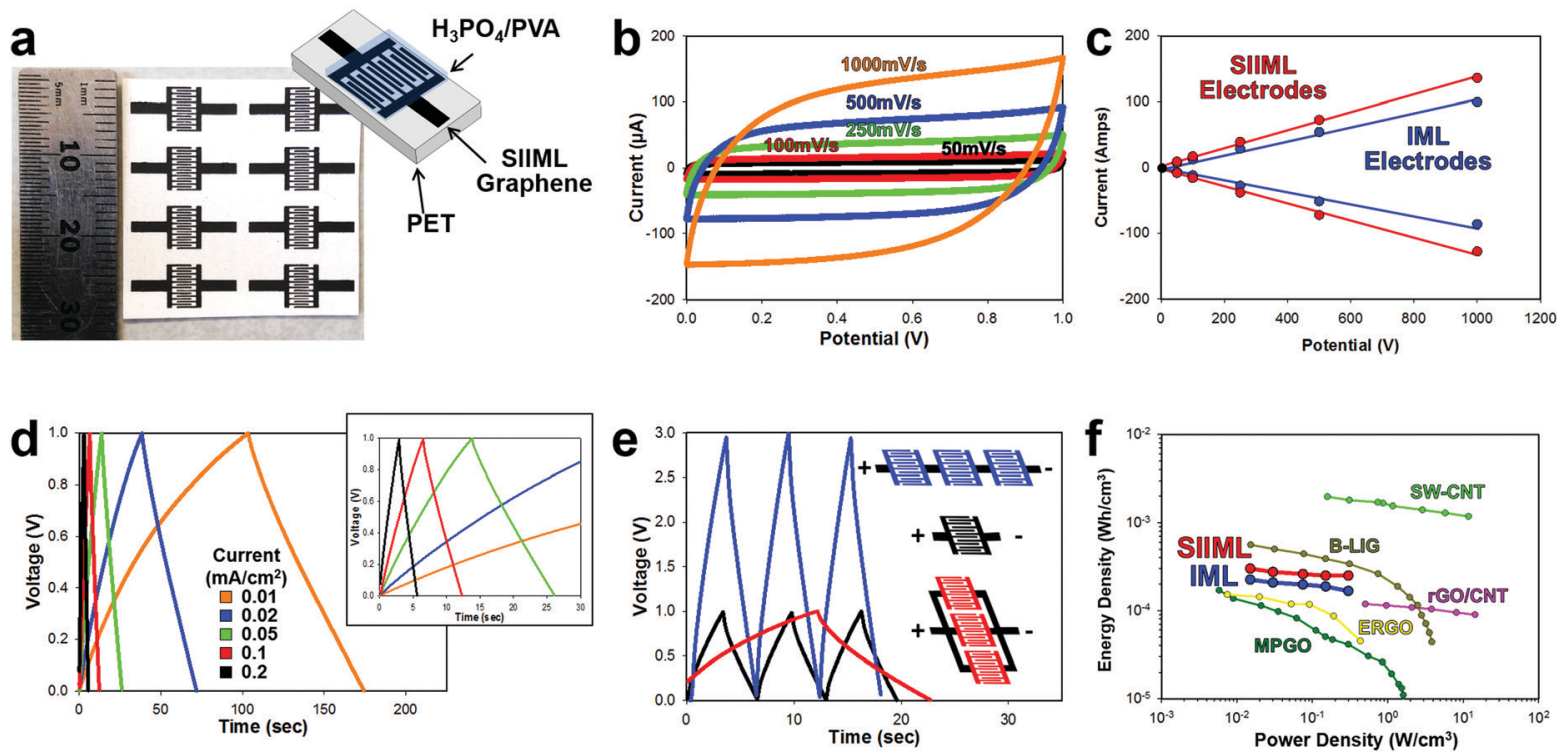

Fig. 7 Electrochemical performance of a SIIML supercapacitor. (a) Photograph of patterned SIIML graphene electrode with schematic of supercapacitor design. (b) Cyclical voltammograms with increasing scan rates $\left(50 \mathrm{mV} \mathrm{s}^{-1}\right.$ black to $1000 \mathrm{mV} \mathrm{s}^{-1}$ orange). (c) Scan rate versus current demonstrating linear relationship and enhanced capacitance of SIIML (red) over IML (blue). (d) Galvanostatic charge-discharge curves with different current densities $\left(0.01 \mathrm{~mA} \mathrm{~cm} \mathrm{~cm}^{-2}\right.$ - orange to $0.2 \mathrm{~mA} \mathrm{~cm}-2$ red), inset: magnification showing near triangular shapes at higher current densities. (e) Galvanostatic charge-discharge graph of single supercapacitor (black), three electrodes connected in series (blue), and three electrodes connected in parallel (red). (f) Ragone plot of SIIML (red) and IML (blue) supercapacitor electrodes and various other carbon-based IDE supercapacitors; light green: inkjet printed single walled carbon nanotubes (SW-CNT), ${ }^{63}$ brown: boron doped laser induced graphene (B-LIG), ${ }^{69}$ pink: electrostatic spray deposited reduced graphene oxide/carbon nanotube (rGO/CNT), ${ }^{70}$ yellow: electrochemically reduced graphene oxide (ERGO), ${ }^{71}$ and dark green: methane plasma treated graphene oxide with gold collector (MPGO) ${ }^{72}$ 
internal resistance. The capacitive current (due to double layer charging) of the SIIML graphene electrodes was extracted from the CVs and compared with IML graphene with no salt. The graphene with salt pores displayed enhanced double layer capacitance (37\%) current as portrayed in Fig. 7c. Galvanostatic charge-discharge profiles of the SIIML were then graphed which shows relatively symmetric triangular shape, but slowly loses symmetry at very low charging densities $\left(0.01 \mathrm{~mA} \mathrm{~cm}{ }^{-2}\right)$. Based on the discharge rates, the capacitance was calculated and graphed versus the current density, Fig. S8 (ESI $\dagger$ ). On average, the SIIML graphene had $\sim 30 \%$ more capacitance than the devices without salt (IML).

Multiple supercapacitors can be connected in series (to boost voltage capabilities) or in parallel (to increase energy storage density) as illustrated and graphed in Fig. 7e. When three electrodes are connected in series (blue) the voltage window increased by a factor of three with roughly the same charge-discharge times. In contrast, when three electrodes are connected in parallel, the charge-discharge times increase by a factor of three with the voltage window remaining constant. Therefore, these supercapacitors could easily be patterned in series or parallel to adapt for a specific application. To demonstrate that SIIML graphene patterning process for supercapacitor use, an array of supercapacitors were fabricated ( 3 in series and 5 in parallel) and were used to power a red LED (drop voltage $\sim 1.8 \mathrm{~V}$ ). After charging the supercapacitor to $3 \mathrm{~V}$, the LED was tested across the supercapacitor array and illuminated multiple times before the supercapacitor's voltage decreased below $1.8 \mathrm{~V}$, see Movie S3 (ESI $\dagger$ ).

Finally, the SIIML graphene and IML graphene (no salt) energy density and power density were extracted from the galvanostatic discharge and graphed on a Ragone plot (Fig. 7f). ${ }^{63,64}$ The graphene electrodes that contained salt pores demonstrated higher energy density due to the increase in ECSA which stored more charge at the graphene/electrolyte interface. Both the SIIML and the IML graphene interdigitated electrodes compared favorably with other carbon-based supercapacitors IDEs reported in the literature, with the SIIML outperforming the IML electrode

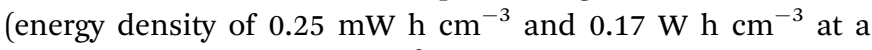
power density of $0.3 \mathrm{~W} \mathrm{~cm}^{-3}$, respectively). Note that supercapacitors are strongly dependent on the electrolyte used, and by using a more efficient electrolyte, a higher energy and power density could be obtained. ${ }^{65-68}$

\section{Conclusions}

In conclusion, a micromanufacturing technique for enhancing the electrocatalytic activity of patterned solution-phase graphene was demonstrated with the use of a modified IML technique. This technique creates a multiscale porous architecture in the graphene surface. Macrosized pores $(25-50 \mu \mathrm{m})$ are formed by utilizing salt crystals as porogens (hard templates) in the graphene ink while patterning, referred to as salt impregnated inkjet maskless lithography (SIIML). Additionally, microsized pores $(100 \mathrm{~nm}-2 \mu \mathrm{m})$ are etched into the graphene surface through $\mathrm{CO}_{2}$ laser annealing, even on flexible heat sensitive substrates (PET). We demonstrate that SIIML is an effective tool for enhancing the electrochemical activity of graphene by fabrication of an electrochemical pesticide biosensor, which utilizes ACHE. Graphene electrodes with macrosized pores through salt impregnation outperformed their non macropore counterparts (sensitivity to ACTH of $28.3 \mu \mathrm{AnM}^{-1}$ to $13.3 \mu \mathrm{AnM}^{-1}$ ). The final SIIML graphene ACHE sensor had a wide linear sensing range (10 $\mathrm{nM}$ to $500 \mathrm{nM})$, low limit of detection $(0.6 \mathrm{nM})$, and high sensitivity (12.4 $\mathrm{nA} \mathrm{nM}{ }^{-1}$ ) to paraoxon which is below the tolerable drinking limit reported by the EPA. ${ }^{53}$ Furthermore, we demonstrate that this method can fabricated complex geometries such as interdigitated electrodes for use in other non-biosensing related applications (i.e., an electrochemical supercapacitor). Critically, the SIIML graphene outperformed non-macroporous graphene by demonstrating $\sim 30 \%$ higher capacitance. The SIIML supercapacitor demonstrated excellent energy density of $0.25 \mathrm{~mW} \mathrm{~h} \mathrm{~cm}{ }^{-3}$ at a power density of $0.3 \mathrm{~W}$ $\mathrm{cm}^{-3}$, which was comparable to other carbon-based supercapacitors reported in literature. Both the electrochemical biosensor and supercapacitor demonstrate that increasing the electrochemical surface area of solution-phase printed graphene electrodes via SIIML and $\mathrm{CO}_{2}$ laser annealing improves the performance of these electrochemical devices. Moreover, these manufacture techniques (SIIML and $\mathrm{CO}_{2}$ laser annealing) are amenable to thermally sensitive and flexible substrate materials; therefore, this method could be adapted to wearable technology for energy storage or textile-based biosensors ${ }^{5,73-75}$ as well as for potential creation of flexible supercapacitors on paper-based substrates. ${ }^{76}$ Finally, this high ECSA graphene transduction electrode can act as a platform technology for additional electrochemical sensors or supercapacitors, as well other electrochemical devices such as batteries, ${ }^{77}$ biofuel cells, ${ }^{78}$ dye sensitive solar cells, ${ }^{79}$ or electrode stimuli. ${ }^{8,48}$

\section{Conflicts of interest}

The authors declare no competing financial interest.

\section{Acknowledgements}

J. C. C. gratefully acknowledges funding support for this work by the National Institute of Food and Agriculture, U.S. Department of Agriculture, under award number 2018-67011-27989 and 201667021-25038, from the National Science Foundation under award number CBET-1706994 and ECCS-1841649, and the Roy J. Carver Trust under award number 15-4615. I. L. M. acknowledges ONR and NRL for Funding Support. Special thanks is given to Steven Kmiec and Steve Martin of Materials Science and Engineering (MSE) of Iowa State University for providing Raman Spectra.

\section{References}

1 R. Raccichini, A. Varzi, S. Passerini and B. Scrosati, Nat. Mater., 2015, 14, 271.

2 S. L. Burrs, M. Bhargava, R. Sidhu, J. Kiernan-Lewis, C. Gomes, J. C. Claussen and E. S. McLamore, Biosens. Bioelectron., 2016, 85, 479-487. 
3 F. Bonaccorso, L. Colombo, G. Yu, M. Stoller, V. Tozzini, A. C. Ferrari, R. S. Ruoff and V. Pellegrini, Science, 2015, 347, 1246501.

4 H. Yang, S. Kannappan, A. S. Pandian, J.-H. Jang, Y. S. Lee and W. Lu, Nanotechnology, 2017, 28, 445401.

5 K. Share, A. P. Cohn, R. Carter, B. Rogers and C. L. Pint, ACS Nano, 2016, 10, 9738-9744.

6 M. Liu, R. Zhang and W. Chen, Chem. Rev., 2014, 114, 5117-5160.

7 M. Uz, S. R. Das, S. Ding, D. S. Sakaguchi, J. C. Claussen and S. K. Mallapragada, Adv. Healthcare Mater., 2018, 1701046.

8 S. R. Das, M. Uz, S. Ding, M. T. Lentner, J. A. Hondred, A. A. Cargill, D. S. Sakaguchi, S. Mallapragada and J. C. Claussen, Adv. Healthcare Mater., 2017, 6, 1601087.

9 S. R. Das, Q. Nian, A. A. Cargill, J. A. Hondred, S. Ding, M. Saei, G. J. Cheng and J. C. Claussen, Nanoscale, 2016, 8, 15870-15879.

10 J. A. Hondred, J. C. Breger, N. J. Alves, S. A. Trammell, S. A. Walper, I. L. Medintz and J. C. Claussen, ACS Appl. Mater. Interfaces, 2018, 10, 11125-11134.

11 J. Pang, R. G. Mendes, P. S. Wrobel, M. D. Wlodarski, H. Q. Ta, L. Zhao, L. Giebeler, B. Trzebicka, T. Gemming, L. Fu, Z. Liu, J. Eckert, A. Bachmatiuk and M. H. Rümmeli, ACS Nano, 2017, 11, 1946-1956.

12 W. Yuan, Y. Zhou, Y. Li, C. Li, H. Peng, J. Zhang, Z. Liu, L. Dai and G. Shi, Sci. Rep., 2013, 3, 2248.

13 R. K. Paul, S. Badhulika, N. M. Saucedo and A. Mulchandani, Anal. Chem., 2012, 84, 8171-8178.

14 C. X. Cong, T. Yu, Z. H. Ni, L. Liu, Z. X. Shen and W. Huang, J. Mater. Chem. C, 2009, 113, 6529-6532.

15 Z. Fan, Q. Zhao, T. Li, J. Yan, Y. Ren, J. Feng and T. Wei, Carbon, 2012, 50, 1699-1703.

16 X. Dong, X. Wang, L. Wang, H. Song, H. Zhang, W. Huang and P. Chen, ACS Appl. Mater. Interfaces, 2012, 4, 3129-3133.

17 B. G. Choi, M. Yang, W. H. Hong, J. W. Choi and Y. S. Huh, ACS Nano, 2012, 6, 4020-4028.

18 K. Olszowska, J. Pang, P. S. Wrobel, L. Zhao, H. Q. Ta, Z. Liu, B. Trzebicka, A. Bachmatiuk and M. H. Rummeli, Synth. Met., 2017, 234, 53-85.

19 A. A. Green and M. C. Hersam, Nano Lett., 2009, 9, 4031-4036.

20 D. Li, W.-Y. Lai, Y.-Z. Zhang and W. Huang, Adv. Mater., 2018, 30, 1704738.

21 H. Ma, P. Gao, D. Fan, B. Du, J. Hao and Q. Wei, New J. Chem., 2013, 37, 1307-1311.

22 S. H. Lee, H. W. Kim, J. O. Hwang, W. J. Lee, J. Kwon, C. W. Bielawski, R. S. Ruoff and S. O. Kim, Angew. Chem., 2010, 122, 10282-10286.

23 J. L. Vickery, A. J. Patil and S. Mann, Adv. Mater., 2009, 21, 2180-2184.

24 Q. He, S. R. Das, N. T. Garland, D. Jing, J. A. Hondred, A. A. Cargill, S. Ding, C. Karunakaran and J. C. Claussen, ACS Appl. Mater. Interfaces, 2017, 9, 12719-12727.

25 E. Jabari and E. Toyserkani, Carbon, 2015, 91, 321-329.

26 W. J. Hyun, E. B. Secor, M. C. Hersam, C. D. Frisbie and L. F. Francis, Adv. Mater., 2015, 27, 109-115.
27 E. B. Secor, T. Z. Gao, A. E. Islam, R. Rao, S. G. Wallace, J. Zhu, K. W. Putz, B. Maruyama and M. C. Hersam, Chem. Mater., 2017, 29, 2332-2340.

28 B. G. Choi and H. S. Park, J. Phys. Chem. C, 2012, 116, 3207-3211.

29 E. B. Secor, M. H. Dos Santos, S. G. Wallace, N. P. Bradshaw and M. C. Hersam, J. Phys. Chem. C, 2018, 122, 13745-13750.

30 J. Li, V. Mishukova and M. Östling, Appl. Phys. Lett., 2016, 109, 123901.

31 J. A. Hondred, L. R. Stromberg, C. L. Mosher and J. C. Claussen, ACS Nano, 2017, 11, 9836-9845.

32 P. Trogadas, V. Ramani, P. Strasser, T. F. Fuller and M. O. Coppens, Angew. Chem., Int. Ed., 2016, 55, 122-148.

33 B. Zhao and M. M. Collinson, Chem. Mater., 2010, 22, 4312-4319.

34 Y. S. Nam, J. J. Yoon and T. G. Park, J. Biomed. Mater. Res., 2000, 53, 1-7.

35 J. Zhang, L. Wu, D. Jing and J. Ding, Polymer, 2005, 46, 4979-4985.

36 X. Mu, T. Bertron, C. Dunn, H. Qiao, J. Wu, Z. Zhao, C. Saldana and H. J. Qi, Mater. Horiz., 2017, 4, 442-449.

37 X. Shi, B. Sitharaman, Q. P. Pham, F. Liang, K. Wu, W. E. Billups, L. J. Wilson and A. G. Mikos, Biomaterials, 2007, 28, 4078-4090.

38 S. E. Skrabalak and K. S. Suslick, J. Am. Chem. Soc., 2006, 128, 12642-12643.

39 I. V. Pavlidis, M. Patila, U. T. Bornscheuer, D. Gournis and H. Stamatis, Trends Biotechnol., 2014, 32, 312-320.

40 M. Kesik, F. E. Kanik, J. Turan, M. Kolb, S. Timur, M. Bahadir and L. Toppare, Sens. Actuators, B, 2014, 205, 39-49.

41 Y. Yang, H. Fei, G. Ruan, C. Xiang and J. M. Tour, Adv. Mater., 2014, 26, 8163-8168.

42 J. C. Claussen, M. S. Artiles, E. S. McLamore, S. Mohanty, J. Shi, J. L. Rickus, T. S. Fisher and D. M. Porterfield, J. Mater. Chem., 2011, 21, 11224-11231.

43 A. J. Bard and L. R. Faulkner, Fundamentals and Applications, Wiley, New York, 2001.

44 J. C. Claussen, A. Kumar, D. B. Jaroch, M. H. Khawaja, A. B. Hibbard, D. M. Porterfield and T. S. Fisher, Adv. Funct. Mater., 2012, 22, 3399-3405.

45 C. Fernández-Sánchez, E. Pellicer, J. Orozco, C. JiménezJorquera, L. M. Lechuga and E. Mendoza, Nanotechnology, 2009, 20, 335501.

46 V. A. Pedrosa, S. Paliwal, S. Balasubramanian, D. Nepal, V. Davis, J. Wild, E. Ramanculov and A. Simonian, Colloids Surf., B, 2010, 77, 69-74.

47 J. V. Staros, R. W. Wright and D. M. Swingle, Anal. Biochem., 1986, 156, 220-222.

48 S. R. Das, M. Uz, S. Ding, M. T. Lentner, J. A. Hondred, A. A. Cargill, D. S. Sakaguchi, S. Mallapragada and J. C. Claussen, Adv. Funct. Mater., 2017, 6, 1601087.

49 Y. Hernandez, V. Nicolosi, M. Lotya, F. M. Blighe, Z. Sun, S. De, I. T. McGovern, B. Holland, M. Byrne and Y. K. Gun'Ko, Nat. Nanotechnol., 2008, 3, 563.

50 F. R. Wong, A. A. Ali, K. Yasui and A. M. Hashim, Nanoscale Res. Lett., 2015, 10, 233. 
51 N. F. M. Rodrigues, S. Y. Neto, R. D. C. S. Luz, F. S. Damos and H. Yamanaka, Biosensors, 2018, 8, 16.

52 M.-P. Bucur, B. Bucur and G.-L. Radu, Sensors, 2013, 13, 1603-1613.

532018 Edition of the Drinking Water Standards and Health Advisories, DOI: https:/www.epa.gov/sites/production/files/ 2018-03/documents/dwtable2018.pdf.

54 Parathion, DOI: Government of Canada Publications http:// publications.gc.ca/Collection/H48-10-1-64-1991E.pdf.

55 G. Liu and Y. Lin, Anal. Chem., 2006, 78, 835-843.

56 N. Chauhan and C. S. Pundir, Anal. Chim. Acta, 2011, 701, 66-74.

57 D. Du, S. Chen, J. Cai and A. Zhang, Biosens. Bioelectron., 2007, 23, 130-134.

58 X. Sun and X. Wang, Biosens. Bioelectron., 2010, 25, 2611-2614.

59 V. B. Kandimalla and H. Ju, Chem. - Eur. J., 2006, 12, 1074-1080.

60 K. A. Law and S. P. J. Higson, Biosens. Bioelectron., 2005, 20, 1914-1924.

61 M. F. El-Kady and R. B. Kaner, Nat. Commun., 2013, 4, 1475.

62 H.-J. Choi, S.-M. Jung, J.-M. Seo, D. W. Chang, L. Dai and J.-B. Baek, Nano Energy, 2012, 1, 534-551.

63 B. Chen, Y. Jiang, X. Tang, Y. Pan and S. Hu, ACS Appl. Mater. Interfaces, 2017, 9, 28433-28440.

64 A. J. Bard, L. R. Faulkner, J. Leddy and C. G. Zoski, Electrochemical methods: fundamentals and applications, Wiley, New York, 1980.

65 W. Zuo, R. Li, C. Zhou, Y. Li, J. Xia and J. Liu, Adv. Sci., 2017, 4, 1600539.
66 M. Galiński, A. Lewandowski and I. Stępniak, Electrochim. Acta, 2006, 51, 5567-5580.

67 M. D. Stoller, S. Park, Y. Zhu, J. An and R. S. Ruoff, Nano Lett., 2008, 8, 3498-3502.

68 J. Huang, B. G. Sumpter and V. Meunier, Chemistry, 2008, 14, 6614-6626.

69 Z. Peng, R. Ye, J. A. Mann, D. Zakhidov, Y. Li, P. R. Smalley, J. Lin and J. M. Tour, ACS Nano, 2015, 9, 5868-5875.

70 M. Beidaghi and C. Wang, Adv. Funct. Mater., 2012, 22, 4501-4510.

71 B. Xie, C. Yang, Z. Zhang, P. Zou, Z. Lin, G. Shi, Q. Yang, F. Kang and C.-P. Wong, ACS Nano, 2015, 9, 5636-5645.

72 Z. S. Wu, K. Parvez, X. Feng and K. Müllen, Nat. Commun., 2013, 4, 2487.

73 R. K. Mishra, A. Martin, T. Nakagawa, A. Barfidokht, X. Lu, J. R. Sempionatto, K. M. Lyu, A. Karajic, M. M. Musameh and I. L. Kyratzis, Biosens. Bioelectron., 2018, 101, 227-234.

74 M. C. Chuang, J. R. Windmiller, P. Santhosh, G. V. Ramírez, M. Galik, T. Y. Chou and J. Wang, Electroanalysis, 2010, 22, 2511-2518.

75 K. Jost, D. Stenger, C. R. Perez, J. K. McDonough, K. Lian, Y. Gogotsi and G. Dion, Energy Environ. Sci., 2013, 6, 2698-2705.

76 Y.-Z. Zhang, Y. Wang, T. Cheng, W.-Y. Lai, H. Pang and W. Huang, Chem. Soc. Rev., 2015, 44, 5181-5199.

77 H. Kim, K.-Y. Park, J. Hong and K. Kang, Sci. Rep., 2014, 4, 5278.

78 C. Liu, S. Alwarappan, Z. Chen, X. Kong and C.-Z. Li, Biosens. Bioelectron., 2010, 25, 1829-1833.

79 N. G. Sahoo, Y. Pan, L. Li and S. H. Chan, Adv. Mater., 2012, 24, 4203-4210. 